\title{
Are Socio-Econo-Physical Models Better to Explain Biases in Societies?
}

\author{
Soumyajyoti Biswas, ${ }^{* \S}$ F. W. S. Lima ${ }^{\dagger, \pi}$ and Ophir Flomenbom ${ }^{\star * \|}$ \\ *Max Planck Institute for Dynamics and Self-Organization \\ Am Fassberg 17, 37077 Goettingen, Germany \\ ${ }^{\dagger}$ Dietrich Stauffer Computational Physics Lab, Departamento de Fisica \\ Universidade Fereral do Piaui, Teresina, Brazil \\ ‡Flomenbom-BPS Ltd., 19 Louis Marshal Tel Aviv 62668, Israel \\ §soumyajyoti.biswas@ds.mpg.de \\ ॠfwslima@gmail.com \\ "ophir1974@flomenbom.net \\ Received 28 February 2018 \\ Accepted 27 June 2018 \\ Published 13 September 2018
}

\begin{abstract}
In this work, we review various dynamical models in socio-econo-physics, and discuss their roles in understanding socio-economic biases. We deal with fairness in nations, wealth distributions in nations, elections' dynamics and tax evasion models. We claim that these models can better explain biases in societies than traditional models of economics from 1700s that are based on various assumptions such as the "invisible hand concept" that justifies interventions from social beneficiaries.
\end{abstract}

Keywords: Econophysics; sociophysics; opinion dynamics; tax evasion; Gini coefficient.

\section{Introduction}

In many countries around the world, socio-economic inequalities are often coupled to directed socio economic biases. ${ }^{1-9}$ From political biases, banking biases to the "planned obsolesce" practices (where manufacturers produce products that need to be discarded after a certain period ${ }^{10}$ ), the beneficiary groups attempt to keep a bias against the rest, as was manifested in the 2007 crises. The "measures" taken by the political elite arguably made the situation more distorted delivering more powers to the top 1\%. ${ }^{11}$ Recent studies about the USA government decisions showed that the tendency of bias is more general, in that the government often acts in favor of the top $1 \%$. $^{12,13}$

This is an Open Access article published by World Scientific Publishing Company. It is distributed under the terms of the Creative Commons Attribution 4.0 (CC-BY) License. Further distribution of this work is permitted, provided the original work is properly cited. 
The main problems of such biases are seen in the distorted wealth densities (the relative wealth of each socio-economic fraction in the society) and the means by which such distorted wealth densities are created, together with the distortions in the flow of wealth ${ }^{1,14-17}$ and the creation of unequal opportunities. These are global issues that affect various nations.

The economics studies from the very beginning of the $1700 \mathrm{~s}^{18,19}$ were based on the concepts that would justify behind-the-scene interventions to favor the beneficiary group in society. They either ignored the ruling elite's interests to control the wealth (the physiocrats) or used terms like the "invisible hand concept" that clearly justify such interventions.

Models from socio-econo-physics, on the other hand, are based on phenomenological observations and attempt to reproduce such observations in a complex society from emergent cooperativity through minimalistic agent-based modeling. Therefore, this can be a useful tool to understand, underline and explore ways to fight the above-mentioned biases in order to create more fair and stable societies. The solutions should show how to increase the flow of opportunities and ways to generate better wealth densities by abolishing unjust biases.

Given its very broad perspective, here we choose only to address the issue with three models. In this paper we review three models of socio-econo-physics and discuss their roles in explaining the biases in societies. In Sec. 2 society-decider model is reviewed that predicts stability of a society in a crisis and the corresponding wealth distributions. In Secs. 3 and 4 election dynamics and tax evasion models are reviewed that respectively deal with balance between resource allocation and population dynamics, and fair and efficient ways to deal with tax evasion.

\section{The Society Deciders Model, Fairness in Nation and Fair Wealth Distribution}

\subsection{The society-deciders model}

The society-deciders model ${ }^{1}$ (a Lotka-Voltera type model ${ }^{20-22}$ ) can supply answers about and help in explaining what are the economic and sociological stability issues in nations. The model has two groups that are interconnected, the deciders (the top $1 \%$ ) and the society (the rest 99\%) (Fig. 1).

The equations of motion are Langevin-type for the society's temperature $T$ and master equations for the deciders. The society's temperature $T$ appears in the parameters of the master equation, where the opinion computed from the master equation appears in the Langevin equation for $T$. These form the connection between the society and the deciders.

\subsubsection{The society}

$T(t)$ in the society deciders model, the society is defined with the temperature,

$$
T(t)=w(t)+\xi(t)
$$




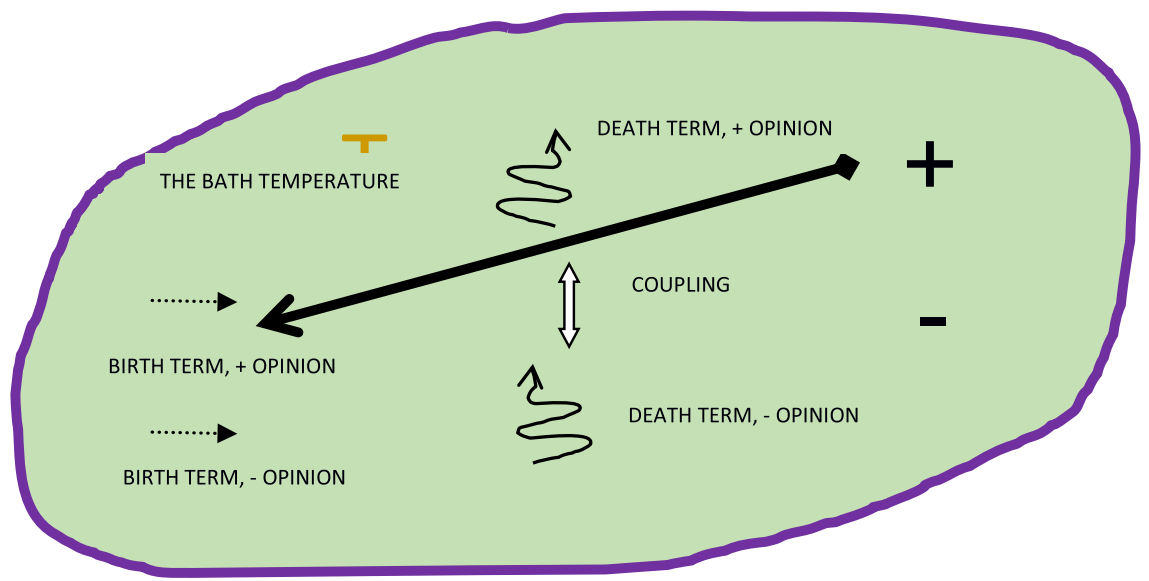

Fig. 1. A schematic illustration of thesociety-deciders model. The whole structure is representing the nation: the society and the deciders; within the structure, the "bulk" is thesociety, represented with a temperature $T$, and the society-top, the "deciders", are represented through the potentials' curves (the inclined curves), where thearrows show deciders flow [either from the system (curly arrows) or between opinions, or inside the system (broken dashed)].

wherein $w(t)$ is the total opinion of the deciders, see next, $\xi(t)$ is a Brownian motion with variance $c$ that stands for all the occurrences in the nation of interest and in the other nations that have an effect on the system that is being tested. $T$ can take any real value. When $|T(t)|$ is small, the society is stable, while it is in a crisis if $|T(t)|$ is large.

\subsubsection{The deciders}

In the society deciders model, the deciders try to have higher influence in their camp, and keep their position, namely surviving. Holding an unpopular opinion for a long time, they risk elimination. The model for the deciders is an equation of motion for $X_{\delta}(x, t)$, the number of deciders, who hold the opinion $\delta$ with a particular level of influence $x$ at time $t$ The $\delta$ is either plus or minus: $\delta=+,-$; the dynamics of $X_{\delta}(x, t)$ is as follows:

$$
\begin{aligned}
\partial_{t} X_{\delta}= & \left(D \partial_{x x}+v \partial_{x}\right) X_{\delta}+k_{b, \delta} \delta(x)-\left(k_{d, \delta}(x)+f_{\delta}(x)\right) X_{\delta} \\
& -c_{\delta} X_{\delta} \tilde{X}_{-\delta}+c_{-\delta} X_{-\delta} \tilde{X}_{\delta} \\
\delta= & +,-
\end{aligned}
$$

The deciders diffuse in the influence coordinate $x\left(D \partial_{x x}\right)$, where a constant force that attracts towards the origin exists $\left(v \partial_{x}\right)$, showing that it is hard to win influence in the deciders' system. At the origin there is a reflecting boundary. Birth $\left(k_{b, \delta} \delta(x)\right)$ and death terms $\left(-k_{d, \delta}(x)\right)$ are included. Deciders join the system at the origin. The death term represents deciders' elimination. The death and the birth terms are balanced most of the time, and only in extreme conditions a net change in the number of deciders is observed. Terms for changes in the opinions are also there in 
Eq. (2), wherein a stress term $\left(f_{\delta}(x)\right)$ represents those that decided to start from scratch with a different opinion. A reaction represents those that decided switching groups after encountering with deciders of at least a similar influence yet within a different opinion $\left(-c_{\delta} X_{\delta} \tilde{X}_{-\delta}\right)$. In this situation, the decider switches opinion yet would still be having the exact level of influence that was obtained during the previous years, which were spent in the other group. All the functions that control the dynamics and survival of the deciders depend on $e^{\delta \frac{T}{\bar{T}}}$, making the connection among the deciders and the people. $\tilde{T}$ is therefore the interaction strength among the society and the deciders. The index fairness is the $\tilde{T}$ relative to the optimal value. Figure 1 illustrates the process. The opinion $w$ in Eq. (1) is a simple average of the functions of $X_{ \pm}(x, t){ }^{1}$

The system is either stable or collapsing. If the deciders collapse due to harsh conditions in the society camp, the nation is defined as fair. Hence fairness in a nation is indicated by the stability of a nation that favors change for the betterment of its vast majority of citizens.

\subsection{Results from the society-deciders model}

The society deciders model showed that central to all approaches for solving the economic woes in (almost) any nation is shifting the wealth and the control on the flow of wealth in the direction of the vast majority in a society, farther from the deciders, instead of cutting or increasing the governmental spending. Therefore, the model proposes solutions for the profound economic problems rather than simply suggesting "buying time" until the next crisis. This requires applying measures that are increasing fairness in the society (checking the effect of any regulation on the stability of the deciders is therefore crucial).

Within the model, long lasting crises are solved by increasing fairness rather than interventions that increase or decrease the governmental debt. This is a very different result from the traditional economics that always focus the attention in endless debates about increasing or decreasing government's debts, where the proposed solution deals with other issues than governmental debts, for example, the effect of these measures on the distortions in the wealth distributions.

\subsection{Wealth densities}

Wealth densities tell "all stories" of the societies. ${ }^{23-47}$ We write the dynamical equation of the wealth density $f(c, t)$ (the probability density function to have wealth $c$ at time $t$ ) where each term depicts the hidden situation with the hidden assumptions in a society. From $f(c, t)$ we compute the Gini coefficient (it is the departure of the distribution from a uniform distribution). For example, with equation of motion with linear force, the Gini coefficient is

$$
\text { Gini }=\frac{1}{2\left(1+\frac{\mu}{\theta}\right)} \leq 50 \%,
$$

where $\mu$ is the minimal wage and $\mu+\theta$ is the mean wage. This is a basic result in wealth densities, showing that when the society is fair in the sense that rich face 
difficulties to have more money relative to their situation, the exponential steady state wealth density has upper limit of $50 \%$ in the Gini coefficient value. In most cases, the Gini coefficient is smaller than $50 \%$. The Gini coefficient is above $40 \%$ when $\theta \geq 4 \mu$ i.e., the mean wage is five times the minimal wage, indicating severe conditions in such a society (in most cases the mean wages are just twice the minimal wages).

Utilizing the society deciders model to create wealth densities, we find in $^{2}$ that a democratic and a free nation, a fair society is such that the income distribution's Gini coefficient is in the range, $25 \%, 33 \%$ and $34 \%$. The value, Gini $\approx 40 \%$, is indeed the transition from the intermediate stage nation to a biased nation, where Gini $\leq 40 \%$ requires that the income distribution is smooth in the top percentiles (rather than having a jump). This result tells what type of properties the potential terms in the equation of motion of the deciders camp should have. ${ }^{2}$

The society deciders type of models, explicitly built with two types of camps in the society, and can explain societies much better than models that deliberately ignore such structures in societies.

\section{Modeling Politics with Opinion Models}

\subsection{Minority candidate winning situations}

In 2016 U.S. presidential election, one of the surprises was to see the candidate with less number of popular vote winning the presidency. Although rare, this is not the first U.S. election with such an outcome, but this has happened four times $(1876,1888,2000$ and $2016)$ in past 49 elections. This is a direct consequence of the "winner takes all" rule placed in most of the states while assigning electoral college votes. This is a form of a majority rule, where the "local" majority within a state is considered in assigning all the members (except in few states) of the electoral college of a state.

There are other methods used in countries around the world for elections, e.g., the direct vote count, proportional representations, indirect elections, etc. There are also parliamentary democracies, where the leader is elected through the support of the elected members of the parliament. Each of these methods has its own justifications in terms of fairness (resource allocation, etc.) of the representation of the people's will.

Here we shall be considering the "winner takes all" method used in the U.S. The fundamental questions in that situation are the following: (a) when does the overall majority not coincide with the majority of the electoral college and how to quantify that? (b) how does these reflect upon the fairness of the processes and acceptability among the general population (in this context, it is worth mentioning that the minority win scenario has happened in the past and the voting system remained in place in spite of it)?

\subsection{Equilibrium and nonequilibrium opinion dynamic models}

As mentioned before, the minority-win phenomenon is a result of a local majority rule. Majority rules are used in various contexts in modeling socio-economic 
phenomena, ${ }^{4-51}$ for example the majority voter model, models of tax evasions ${ }^{52}$ (as discussed in Sec. 4), etc. The local majority rule is the fundamental aspect of coarse-graining mechanism as a building block of renormalization group analysis in spin models. While the scale free behavior of a system near criticality ensures the formal equivalence of the Hamiltonian, it does not guarantee the conservation of the overall sign of the spins. However, this is a vital quantity in election results in particular.

the model All agents $(N)$ with opinions $\pm 1,0$, are divided into several groups, where the majority sign in each group is taken as the sign of the whole group (coarse-graining mechanism; see Fig. 2). Following this step the overall sign might get reversed. This is more probable when the initial difference between the two opinions is small (a closely fought election).

As for the dynamics of the opinion values among the agents, in the present case, the opinion of the $i$ th individual at time $t+1$ can be written as $o_{i}(t+1)=o_{i}(t)+$ $\mu_{i j} o_{j}(t)$, where $o_{m} \in+1,0,-1$ and $\mu_{i j}$ can be -1 with probability $p$ and else $+1 .^{53}$ This is similar to, but not same as, the other kinetic exchange models for opinion formation. ${ }^{54-56}$

Previous studies along this line include random partitioning of population and the subsequent conversion of the opinions of all the agents to that of the majority. ${ }^{57-59}$ This process leads an initial minority to win or become majority. In this model, the individual opinion values are not converted. However, a spatial coarse graining is done, which changes the overall sign of the opinion. There were some other attempts that had also been made prior to the 2016 election, ${ }^{59}$ to explain a minority win in a large number of elections, ${ }^{60}$ the deficit of the mean majority with equipopulous units (states), ${ }^{61}$ and also the possibility of having prejudice among the likely voters. ${ }^{59}$

One can now measure in the model, the fraction of times a "minority-win" scenario took place, as a function of the popular vote margin. ${ }^{62} \mathrm{~A}$ more formal quantification is possible in terms of classical information theory. One way to think of the coarse-graining process is transmission of the signal through a noisy channel. ${ }^{63}$

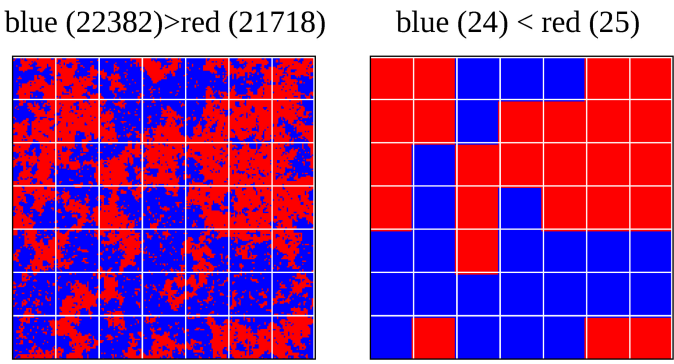

Fig. 2. Snapshots of the spatial organizations of the opinion values prior to (left) and after (right) the coarse graining are shown. Up-spins are in the majority (blue 22382, red 21718) prior to coarse graining, in the original system. But as there is strong spatial correlations, in the coarse-grained system the down spins become the majority (from Ref. 62). 
In this particular case, the input signal is the sequence of the majority sign in the total population at every time step and the output series is again the majority sign, but in the corresponding coarse-grained system. If there were no noise in the "transmission channel", the input and output should always match exactly. However, due to the spatial correlation in the opinion values, there are instances where the overall minority can win after coarse graining, hence giving the "noise" in the channel (see Ref. 62 for details).

Regarding the question of fairness of the reflection of people's will in the final result, it is worth noting that the system of winner takes all is in place to reduce the influence of larger states in the election results. That consideration balances the apparent contradiction with the loss of information measured above in this situation.

We have not considered effect of zealots, who do not change their opinions and the effect of media as a global bias. These are interesting future directs of study.

\section{Tax Evasion}

\subsection{Modeling tax evasion}

Modeling the tax evasion might help improving fairness in societies. Although most unfair tax occurrences are the reality in most nations, where the rich do not pay their fair share of tax and evade tax in "legal" ways, illegal tax evasions also occur and should be dealt with. How to increase tax participation and how to do this in the most efficient way is part of the work reviewed in this section.

\subsection{Zaklan model via nonequilibrium dynamics of Majority-vote Model (MVM)}

The Zaklan model ${ }^{64,65}$ consists of a number of agents located on a regular or irregular structure. In every time period each network site is inhabited by an individual, spin, $\sigma_{i}$ who can either be an honest tax payer $\sigma_{i}=+1$ or a cheater $\sigma_{i}=-1$. Initially it is assumed that everybody is honest. At each step, individuals can rethink their behavior and have the opportunity to become the opposite type of agent they were in the previous step. The network neighborhood of every individual is composed of $z$ people. The agents on social network may either prefer withhold taxes or not. This decision depends on two factors: First, the type of network every agent is connected with exerts influence on what type of citizen the person becomes in the respective period. On the other hand, people's decisions are partly autonomous, i.e., they are not only influenced by the constitution of their vicinity, but can also decide for themselves. If initially honest agents decide to evade taxes, these can influence the others to do so as well. Applied in the case of tax evasion, the model can be interpreted as follows: The tax evaders have the greatest influence to turn honest citizens into tax evaders if they make a majority in the neighborhood of honest citizens. If the majority evades, one is likely to also evade. On the other hand, if most people in the vicinity are honest, the respective individual is likely to become a tax payer if the 
individual was a tax evader before. The model also present the enforcement mechanism that consists of two components: a probability of an efficient audit $p_{a}$; and if tax evasion is detected, the individual must remain honest for subsequent $K$ steps. One time unit is one sweep through the entire lattice. The time evolution of this model can be performed by using an equilibrium or non-equilibrium dynamics. In the MVM on network, the system dynamics traditionally is as follows: We assign a spin variable $\sigma_{i}$ with values \pm 1 at each node of the network. At each step we try to spin flip a node. The flip is accepted with probability

$$
w_{i}=\frac{1}{2}\left(1-(1-2 q) \sigma_{i} \cdot S\left(\sum_{J} \sigma_{J}\right)\right)
$$

where $S(x)$ is the sign of $x$ if $x \neq 0, S(x)=0$ if $x=0$. To calculate $w_{i}$ our sum runs over the $z$ nearest neighbors of spin $i$ on the network. Equation (4) means that with probability $(1-q)$ the spin will adopt the same state as the majority of its neighbors. The control parameter $q(0 \leq q \leq 1)$ plays a role similar to the temperature in equilibrium systems: the smaller $q$, the greater the probability of parallel aligning with the local majority.

In this model an agent has opinion and can assume the value \pm 1 depending on the opinion of the majority of its neighbors.

We further use a probability of an efficient audit $p_{a}$. Therefore, if tax evasion is detected by this audit, the agent must remain honest for subsequent $K$ time steps. Again, one time step is one sweep through the entire network.

The opinion of a people community per total number of people is given by

$$
O(q)=\frac{\sum_{i} \sigma_{i}}{N}
$$

In order to model tax evasion, we further use for all agents one probability $p_{a}$ of an efficient audit. If tax evasion, $\sigma_{i}=-1$ is detected by this audit, the agent must remain honest, $\sigma_{i}=+1$, for a number $K$ of time steps. Again, one time-step is one sweep through the entire network.

To get the control of the tax evasion dynamics we use the fraction of tax evaders given by

$$
\text { taxevasion }=\frac{N-N_{\text {honest }}}{N},
$$

where $N$ is the total number and $N_{\text {honest }}$ the honest number of agents. The tax evasion is calculated at every time to step $t$ of system evolution.

The model describe the tax evasion fluctuation of a community of people in a country and propose tools to control that and to decrease the amount of tax evaders.

Our results show that the mechanism fit the data better than the previous model. ${ }^{65}$ In short it is necessary to analyze more quantitative empirical data to understand how tax evaders react to punishment. For example, some governments 
bought bank account data stolen from Swiss banks, and as a result, lots of people admitted to the tax authorities that they did not report the income from the investments before. Informations on amount of income, amount of punishment and behavior in later years are hidden behind tax secrecy. With such information, we can find hidden patterns in society and develop the most efficient mechanism for dealing with tax evaders, like incorporating the informations in the Potts model with $q$ states $^{66}$ to model the tax evasion rate in a community.

\section{Conclusions}

In this work, we reviewed various socio-economic models regarding fairness in nations. The society-deciders model is reviewed in Sec. 2. This model can check whether a nation is stable in the sense that the situation would likely to be improving in favor of the society in times of a crisis. Modeling of the wealth density based on such model is also reviewed, that can answer questions on how to create less skewed wealth densities that would result in increased fairness in nations.

Elections dynamics and tax evasion models are reviewed in Secs. 3 and 4, respectively. Elections' dynamics can deal with fairness in multi-state nations, answering about the balance between resource allocation and population's distribution. Tax models can deal with physiological effects in tax evasion and how to deal with tax evasion fairly and efficiently. These models all show the diversity and strength of socio-econo-physics models in describing societies; rather than justifying " $1 \%$ economics", socio-economic models can help build better societies that are balanced, fair and stable with better flow of opportunities.

\section{References}

1. O. Flomenbom, Biophys. Rev. Lett. 10 (2015) 157.

2. O. Flomenbom, Rep. Adv. Phys. Sci. 01 (2017) 1740004.

3. Here, the reader is encouraged seeing articles appearing in the media from familiar economists about the structure of $1 \%$ and $99 \%$ : P Krugman We are the $99.9 \%$ in NY Times (2011), and J E Stiglitz Of the 1\%, by the 1\%, for the 1\% in Vanity Fair (2011).

4. G. W. Domhoff, Who Rules America? Challenges to Corporate and Class Dominance (McGraw-Hill Humanities, N.Y., 2009).

5. A. Chatterjee, G. Unal Coban and O. Flomenbom, Rep. Adv. Phys. Sci. 01 (2017) 1703001.

6. O. Flomenbom, G. Unal Coban and Y. Adigüzel, Biophys. Rev. Lett. 11 (2016) 55.

7. O. Flomenbom and J. Mazaj, Rep. Adv. Phys. Sci. 01 (2017) 1771001.

8. D. Colander, M. Goldberg, A. Haas, K. Juselius, A. Kirman, T. Lux and B. Sloth, Crit. Rev. 21 (2009) 249.

9. D. Acemoglu and J. Robinson, Why Nations Fail: The Origins of Power, Prosperity, and Poverty (Profile Books Ltd., London, Great Britain, 2012).

10. https://vimeo.com/162337948.

11. See, "Striking it Richer: The Evolution of Top Incomes in the United States", Emmanuel Saez, a draft that appears in the website of Emmanuel Saez, https://eml.berkeley.edu/ saez/saez-UStopincomes-2013.pdf. 
12. M. Gilens and B. I. Page, Testing theories of american politics: Elites, interest groups, and average citizens, Perspect. Politics, in press (2014).

13. E. Guillaud, J. Econ. Inequal. 11 (2013) 57.

14. M. Fleurbaey and F. Maniquet, A Theory of Fairness and Social Welfare (Cambridge University Press, NY, USA, 2011).

15. D. Herzer and S. Vollmer, J. Econ. Inequal. 10 (2012) 489.

16. T. Piketty, Capital in the Twenty-First Century (The Belknap Press of Harvard University Press, Cambridge, Massachusetts London, England, 2014).

17. E. Guillaud, J. Econ. Inequal. 11 (2013) 57.

18. A. Smith, The Theory of Moral Sentiments (Edinburgh, 1759).

19. F. Quesnay, Tableau économique (Economic Table, 1758).

20. A. J. Lotka, J. Phys. Chem. 14 (1909) 271.

21. A. J. Lotka, Elements of Physical Biology (Williams and Wilkins, Baltimore, Maryland, USA, 1925).

22. V. Volterra, Variations and fluctuations in the number of individuals in animal species living together, in Animal Ecology, eds. R. N. Chapman (McGraw-Hill, New York, 1931).

23. Y. Tao and X. Wu, Rep. Adv. Phys. Sci. 01 (2017) 1740005.

24. Y. Adigüzel, O. Flomenbom and G. Unal Coban, Rep. Adv. Phys. Sci. 1 (2017) 1750001.

25. D. G. Champernowne, The Distribution of Income between Persons (Cambridge University Press, 1973).

26. C. Gini, Concentration and Dependency Ratios (in Italian, 1909), English translation in: Rivista di Politica Economica 87 (1997) 769.

27. K. Xu, How has the Literature on Gini's Index Evolved in the Past 80 Years? Economics working paper, Dalhousie University, http://ssrn.com/abstract $=423200$ or doi:10.2139/ ssrn.423200 (2003).

28. S.-B. Kang and Y.-S. Cho, Korean J. Comput. Appl. Math. 5 (1998) 213.

29. F. Clementi and M. Gallegati, Physica A 330 (2005) 427.

30. A. C. Silva and V. M. Yakovenko, Europhys. Lett. 69 (2005) 304, arXiv: cond-mat/ 0406385 .

31. Y. Tao, Universal Laws of Human Society's Income Distribution, http://arXiv.org/abs/ 1506.05418 .

32. J.-P. Bouchaud and M. Mézard, Physica A 282 (2000) 536.

33. K. H. Ho, F. K. Chow and H. F. Chau, Phys. Rev. E 70 (2004) 066110.

34. A. Chatterjee and P. Sen, Phys. Rev. E 82 (2010) 056117.

35. A. Chakraborti, D. Challet, A. Chatterjee, M. Marsili, Y.-C. Zhang and B. K. Chakrabarti, Phys. Rep. 552 (2015) 1, arXiv:1305.2121.

36. J. Sienkiewicz and J. A. Hołyst, Phys. Rev. E 80 (2009) 036103.

37. D. Lamper, S. D. Howison and N. F. Johnson, Phys. Rev. Lett. 88 (2002) 017902.

38. S. Fortunato and C. Castellano, Phys. Rev. Lett. 99 (2007) 138701.

39. C. M. Bordogna and E. V. Albano, Phys. Rev. E 76 (2007) 061125.

40. A. Chatterjee, M. Mitrović and S. Fortunato, Sci. Rep. 3 (2013) 1049.

41. A. M. Calvão, N. Crokidakis and C. Anteneodo, PLoS ONE 10 (2015) e0137732.

42. R. Lemoy, E. Bertin and P. Jensen, EPL 93 (2011) 38002.

43. A. Arenas, A. Díaz-Guilera, C. J. Pérez and F. Vega-Redondo, Phys. Rev. E 61 (2000) 3466.

44. Y. Tao, Phys. Rev. E 82 (2010) 036118.

45. J. Tenenbaum, D. Horvatić, S. C. Bajić, B. Pehlivanović, B. Podobnik and H. E. Stanley, Phys. Rev. E 82 (2010) 046104.

46. Y. Tao and X. Chen, Chin. Phys. Lett. 29 (2012) 058901. 
47. B. K. Chakrabarti, A. Chakraborti, S. R. Chakravarty and A. Chatterjee, Econophysics of Income and Wealth Distributions (Cambridge University Press, Cambridge, 2013).

48. C. Castellano, S. Fortunato and V. Loreto, Statistical physics of social dynamics, Rev. Mod. Phys. 81 (2009) 591.

49. S. Galam, Sociophysics: A Physicist's Modeling of Psycho-Political Phenomena (Springer, 2012).

50. P. Sen and B. K. Chakrabarti, Sociophysics an Introduction (Oxford University Press, 2013).

51. F. W. S. Lima, Kinetic continuous opinion dynamics model on two types of Archimedean lattices, Front. Phys. 5 (2017) 47.

52. R. M. Brum and N. Crokidakis, Dynamics of tax evasion through an epidemic-like model, Int. J. Mod. Phys. C 28 (2017) 1750023.

53. S. Biswas, A. Chatterjee and P. Sen, Disorder induced phase transition in kinetic models of opinion dynamics, Physica A 391 (2012) 3257.

54. G. Deffuant, D. Neau, F. Amblard and G. Weisbuch, Mixing beliefs among interacting agents, Adv. Complex Sys. 3 (2000) 87.

55. R. Hegselmann and U. Krause, Opinion dynamics and bounded confidence models, analysis, and simulation, J. Art. Soc. Soc. Simul. 5 (2002) 2.

56. G. Toscani, Kinetic models of opinion formation, Commun. Math. Sci. 4 (2006) 481.

57. S. Galam, The Trump phenomenon, an explanation from sociophysics, arXiv:1609.03933 (2016).

58. S. Galam, Majority rule, hierarchical structure and democratic totalism: A statistical approach, J. Math. Psychol. 30 (1986) 426.

59. S. Galam, Minority opinion spreading in random geometry, Eur. Phys. J. B 25 (2002) 403.

60. M. G. Neubauer, M. Schilling and J. Zeitlin, Exploring unpopular presidential elections, arXiv:1206.2683.

61. M. J. Hinich, R. Mickelsen and P. C. Ordeshook, The electoral college vs. a direct vote: Policy bias, reversals and indeterminate outcomes, J. Math. Sociol. 4 (1975) 3.

62. S. Biswas and P. Sen, Phys. Rev. E 96 (2017) 032303.

63. C. E. Shannon, A Mathematical theory of communication, The Bell Syst. Tech. J. 27 (1948) 379.

64. G. Zaklan, F. Westerhoff and D. Stauffer, J. Econ. Interact. Coordination 4 (2008) 1.

65. G. Zaklan, F. W. S. Lima and F. Westerhoff, Physica A 387 (2008) 5857.

66. D. P. Landau and K. Binder, A Guide to Monte Carlo Simulations in Statistical Physics, 2nd edn. (Cambridge University Press, 2005). 